Peer Reviewed Letter openaccess

\title{
White light emitting diode correlated colour temperature influence on image analysis in Sala mango quality assessment
}

\author{
Wan Long Chiong, ${ }^{\mathrm{a}}$ Ahmad Fairuz Omar ${ }^{\mathrm{b}, *}$ and Mohd Zubir MatJafric \\ aschool of Physics, Universiti Sains Malaysia, 11800, Penang, Malaysia \\ bSchool of Physics, Universiti Sains Malaysia, 11800, Penang, Malaysia. E-mail: fairuz_omar@usm.my. \\ ORCID: https://orcid.org/0000-0002-7879-9102 \\ 'School of Physics, Universiti Sains Malaysia, 11800, Penang, Malaysia. ORCID: https://orcid.org/0000-0001-9313-7548
}

\begin{abstract}
Colour analysis is one of the common techniques used in assessing fruits quality, especially for types of fruit that have a natural transformation in peel colour according to the stage of maturity and ripening. Imaging through RGB analysis is a popular method employed in colour analysis of fruit. Colour observed by human or machine vision is highly depended on ambient lighting, whether in its intensity or the hue of the white illumination source. Hence, the objective of this research is to provide some brief experimental findings on the influence of light emitting diode correlated colour temperature in transforming the colour perceived from the image of Sala mango and the RGB algorithm in predicting the fruits' $\mathrm{pH}$ and soluble solid content.
\end{abstract}

Keywords: white LED, correlated colour temperature, image analysis, fruit colour, fruit quality

\section{Introduction}

Colour is a vital parameter in describing the appearance of a fruit. As certain fruit start to ripen, the skin colour gradually changes from green to yellow and even to orange in some cultivars, indicating chemical changes taking place in the fruit. Skin colour is considered as a significant external parameter in determining the quality of mango fruit as it correlates well to physical, chemical and other sensory properties of the fruit. Colour is often used as indicator to observe maturity, not only for mango but also for other fruits such as banana, ${ }^{1}$ pineapple, ${ }^{2}$ olive ${ }^{3}$ and guava. ${ }^{4}$ Another significant feature of fruit ripening is the change in soluble carbohydrates, which mainly consist of sucrose, fructose and glucose. ${ }^{5}$ The total sugar content determines the sweetness of the fruit and this attribute is a vital characteristic in determining fruit quality. ${ }^{6}$ It has been reported that the final eating quality actually can be linked to total soluble solid at the fully ripe stage. ${ }^{7}$ Besides soluble solids content (SSC), acidity, which is due to the presence of organic acids, malic and citric acids found in most ripe fruit, is also an indicator of mango intrinsic quality. ${ }^{8}$ Acidity usually increases in the early growth phase, reaches a peak and declines gradually until harvest. The increase in sweetness and decrease in acidity simultaneously with accumulation of sugars and organic acids are responsible for the

\section{Correspondence}

Ahmad Fairuz Omar (fairuz_omar@usm.my)

Received: 23 August 2018

Revised: 18 September 2018

Accepted: 28 September 2018

Publication: 6 October 2018

doi: 10.1255/jsi.2018.a11

ISSN: 2040-4565

\section{Citation}

W.L. Chiong, A.F. Omar and M.Z. MatJafri, "White light emitting diode correlated colour temperature influence on image analysis in Sala mango quality assessment", J. Spectral Imaging 7, a11 (2018). https://doi. org/10.1255/jsi.2018.a11

(C) 2018 The Authors

This licence permits you to use, share, copy and redistribute the paper in any medium or any format provided that a full citation to the original paper in this journal is given, the use is not for commercial purposes and the paper is not changed in any way. 
taste development of fruit. ${ }^{9}$ Sugar content and acidity have become significant parameters in the determination of fruit internal quality as investigated by researchers. ${ }^{10-15}$ The application of digital cameras and image analysis is increasingly popular in visual inspection of fruit quality, as it is less expensive than other techniques. There are several reports of research that have used RGB (red-green-blue) indices from fruit images to predict the intrinsic quality of the fruit. For example, Abarra et al. ${ }^{16}$ have used the RGB technique to predict firmness and titratable acidity of mango fruit, while Yahaya et al. ${ }^{17}$ and Xia et al. ${ }^{18}$ have used RGB images to predict $\mathrm{pH}$ and SSC of mango and grapes, respectively.

One of the advantages of imaging compared to spectroscopy in the quality evaluation of fruit is its ability to measure spatial colour distribution of the sample rather than the single-point measurement used in typical spectroscopic analysis. The purpose of this work is to determine the influence of white light emitting diode (LED) correlated colour temperature (CCT) on indices of the RGB colour system in image analysis of intact Sala mango and examine its correlation to the intrinsic quality parameters of the fruit (i.e. pH and SSC of the mangoes flesh).

\section{Materials and methods}

Fifty-one Sala mangoes were collected by courtesy of the Federal Agricultural Marketing Authority (Perlis Branch) with the assistance of experts from the Department of Agriculture at an orchard located in Perlis State, Malaysia. After the harvest, the samples were transported to the School of Physics at Universiti Sains Malaysia where the experimental procedures were carried out. All samples were labelled numerically in order and stored in an airconditioned room at $17^{\circ} \mathrm{C}$ and approximately $65 \%$ relative humidity overnight prior to measurements. The acquisition of images of the intact mango samples was performed in a dark room using the fully automatic camera mode of operation. The model of camera used was a Sony Alpha SLT-A55V (Tokyo, Japan). The camera was set approximately at a distance of $50 \mathrm{~cm}$ above the fruit's surface with two LED panels illuminating at $45^{\circ}$ from both directions. Each LED panel contained four white LEDs and each LED was driven by $400 \mathrm{~mA}$ DC current using an Irwin (0-35 V) DC power supply (Braintree, Essex, UK) and several 10 W cement type resistors to control the current. The experimental setup is shown in Figure 1.

The experiment was carried out using white LEDs from Osram Opto Semiconductor with different CCTs of $3500 \mathrm{~K}$ (D35), 4500 K (D45) and 5700K (D57). The characteristics of the white LEDs used in this experiment are listed in Table 1. Images were captured for both sides of the mango surface; one side was used for the calibration dataset and the other side for the validation dataset. The purpose of the validation set is to test the ability of the system to reproduce the RGB indices from
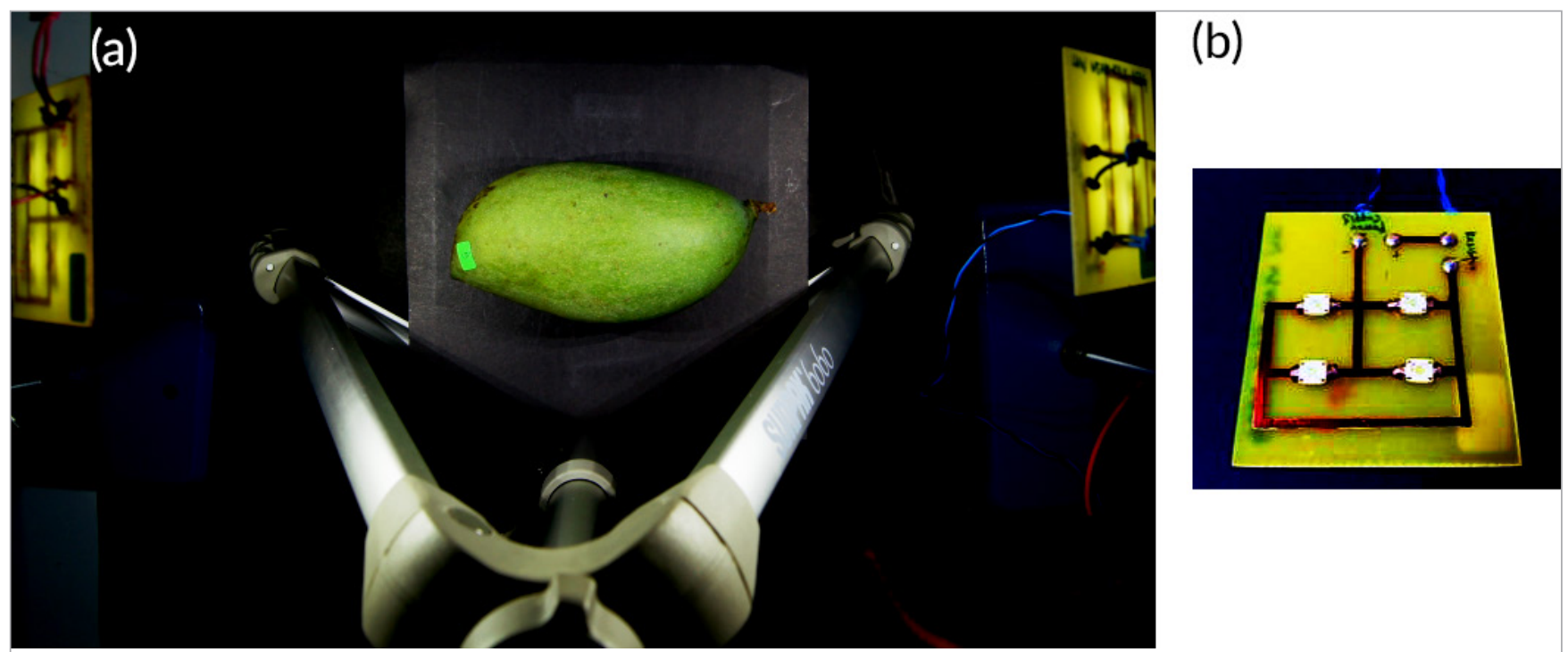

Figure 1. (a) Image of the experimental setup taken from the camera with LED panels located at both sides of the mango sample. (b) Image of an LED panel containing four white LEDs. 
Table 1. Characteristics of individual white LEDs used in this experiment.

\begin{tabular}{|l|l|l|l|}
\hline \multirow{2}{*}{\multicolumn{1}{c|}{ Specifications }} & \multicolumn{2}{c|}{ D35 } & \multicolumn{2}{c|}{ D45 } & \multicolumn{1}{c|}{ D57 } \\
\cline { 2 - 4 } & \multicolumn{1}{c|}{ LCW W5AM-KXKY-4O9Q } & LCW W5AM.PC-KRKT-4J8K & LUW W5AM-KYLX-4E8G \\
\hline Part No. & 3500 & 4500 & 5700 \\
\hline CCT & $26-30 \mathrm{~cd}$ & $93.1 \mathrm{~lm}$ & $26 \mathrm{~cd}$ \\
\hline Luminous intensity & $71-97 \mathrm{Im}$ & $170^{\circ}$ & $106 \mathrm{Im}$ \\
\hline Luminous flux & $170^{\circ}$ & $7.2 \times 6.2 \mathrm{~mm}$ & $170^{\circ}$ \\
\hline Viewing angle & $7.2 \times 6.2 \mathrm{~mm}$ & $7.2 \times 6.2 \mathrm{~mm}$ \\
\hline Dimensions & & \\
\hline
\end{tabular}

the same set of fruit samples under illumination by white LEDs of different CCT. This is done by considering the colour distribution on a single mango sample not to be uniform. The acquired picture files were saved in JPEG format $(4912 \times 2760$ pixels) and were used later for image analysis. Images acquired were imported into the GNU Image Manipulation Program (GIMP) (Version 2.8.14, The GIMP Team) and an area of approximately $1 \mathrm{~cm}^{2}$ at the centre of the mango was specified to obtain RGB colour values of the image. The RGB values represented red, green and blue colour components of an image. The RGB data obtained for a green and yellow-orange sample under different CCT lighting were converted to the CIELAB colour space using the freely available Colormine online converter (http://colormine.org/convert/rgb-tolab). $L^{*} a^{*} b^{*}$ is currently the most used method to represent the colour quantitative description of an object since it can provide more uniform numeric differences regarding visual differences. ${ }^{19,20}$

In this research, reflectance measurement was also conducted to obtain spectra of all white LEDs used in this experiment, in relation to the spectrum of a calibrated tungsten halogen lamp. The reflectance spectra were also taken from two mango samples with distinctive levels of ripeness and peel colour for reference purposes. The reflectance measurement was conducted using a Jaz Spectrometer (Ocean Optics, Dunedin, Florida, USA) in the visible region between $400 \mathrm{~nm}$ and $700 \mathrm{~nm}$. The spectrometer was first calibrated using a white diffuse reflectance standard with tungsten halogen lamp (pro lamp) as the light source, illuminating at $45^{\circ}$ to the white diffuse standard. An optical fibre probe with core diameter of $600 \mu \mathrm{m}$ was placed $1.5 \mathrm{~cm}$ above the white reference to retrieve the reflected light. After the calibration using the tungsten halogen lamp, the light source was replaced with two LED panels and the reflectance meas- urement from the white diffuse reference and the mango samples was taken.

After acquiring the images and the reflectance value, the fruits were peeled and blended using a blender to collect juice for measurement. The $\mathrm{pH}$ value of the fruits was measured using an Exstik pH meter (Extech Instruments, MA, USA) with a range of measurement between $0 \mathrm{pH}$ and $14 \mathrm{pH}$, resolution of $0.01 \mathrm{pH}$ and accuracy of $\pm 0.01 \mathrm{pH}$, while the SSC of the mangoes was obtained using a digital refractometer (PAL-3, Atago Co., Tokyo, Japan) with a range of measurement from $0^{\circ}$ Brix to $93^{\circ}$ Brix, resolution of $0.1^{\circ}$ Brix and accuracy of $\pm 0.2^{\circ}$ Brix. The colour indices obtained were used in the development of predictive algorithms using multiple linear regression (MLR). Different combinations between the $R, G$ and $B$ values were tested to determine the influence of LED CCT on the prediction results. The developed calibration algorithms were then validated using another set of data which were taken from the other side of mango samples.

\section{Results and discussion}

Figure 2 (a) shows the image of two mango samples with different levels of maturity under illumination of white LEDs with different CCT. The green coloured mango in the figure has not reached its full maturity and has a $\mathrm{pH}$ value of 2.92 with SSC of $7.8^{\circ}$ Brix. The yellow-orange mango, on the other hand, is fully ripe with a pH value of 5.24 and SSC of $15.5^{\circ}$ Brix. In general, the colour of a mango changes from dark green to slight yellow and to orange colour due to chlorophyll degradation and increase of the yellow carotenoid pigment. ${ }^{21}$ From visual observation, samples illuminated by lower LED CCT appear darker and sharper in 


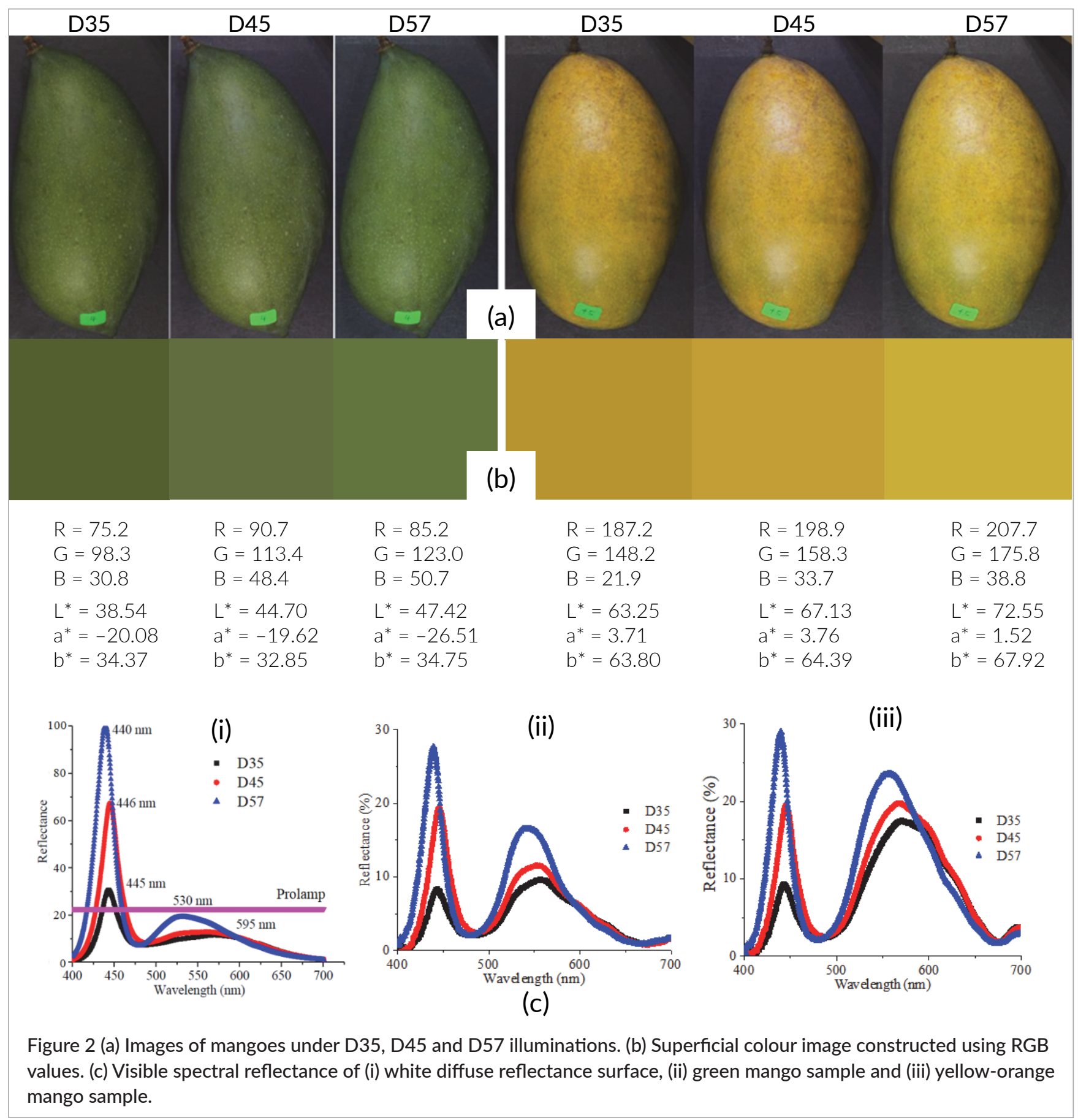

the image compared to samples illuminated by higher LED CCT. In order to enhance the distinctive effect of CCT on the observable colour of the fruits, superficial colour images of the samples were reconstructed using the computed values of RGB as shown in Figure 2(b). Besides obtaining RGB for all samples for quantitative analysis, $L^{*} a^{*} b^{*}$ for mango images in Figure 2(a) were also generated from the respective RGB values. $L^{*} a^{*} b^{*}$ closely represents the colour perceived by the human eye 22 and, hence, was included in this analysis to describe the colour transformation of the fruits under different lighting and different stage of ripeness. Higher $L^{*}$ indicates brighter colour, negative $a^{*}$ indicates green, while positive $a^{*}$ indicates red. On the other hand, negative $b^{*}$ indicates blue while positive $b^{*}$ indicates yellow. Based on this concept, for the green mango, higher LED CCT enhances the green and lighter appearance of the sample. The same observation was 


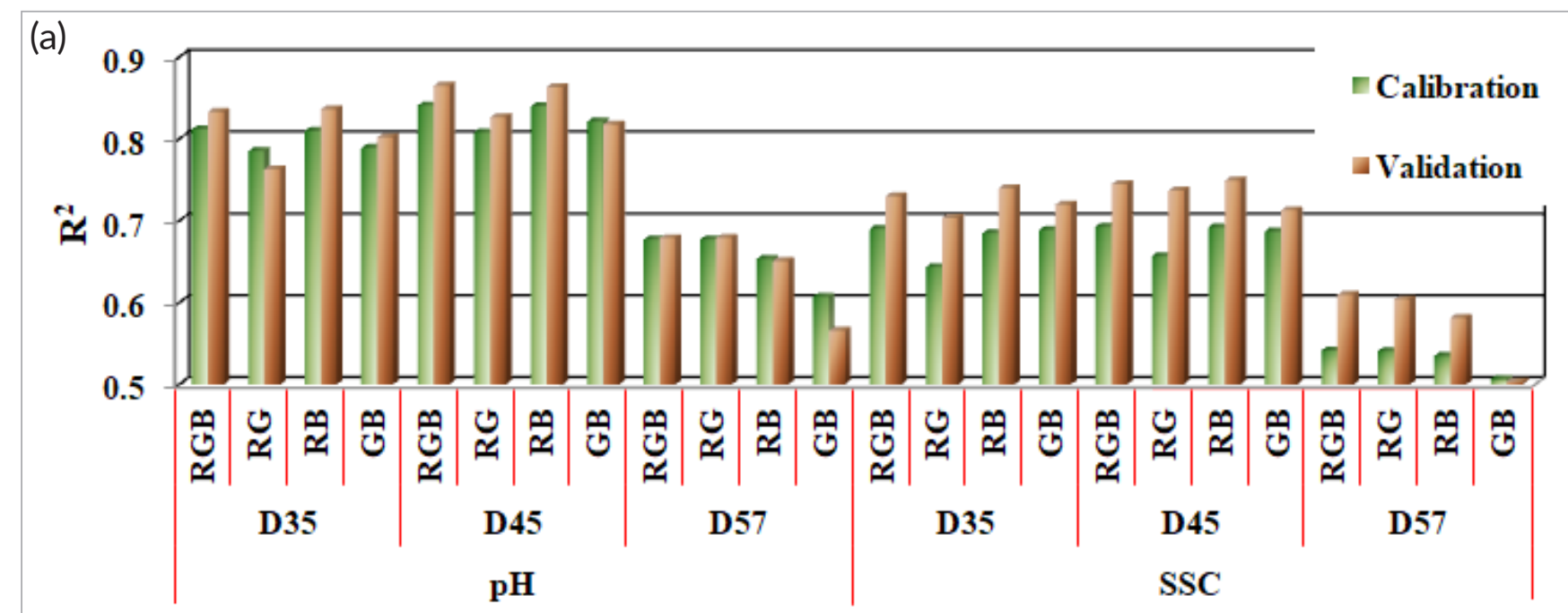

(b)
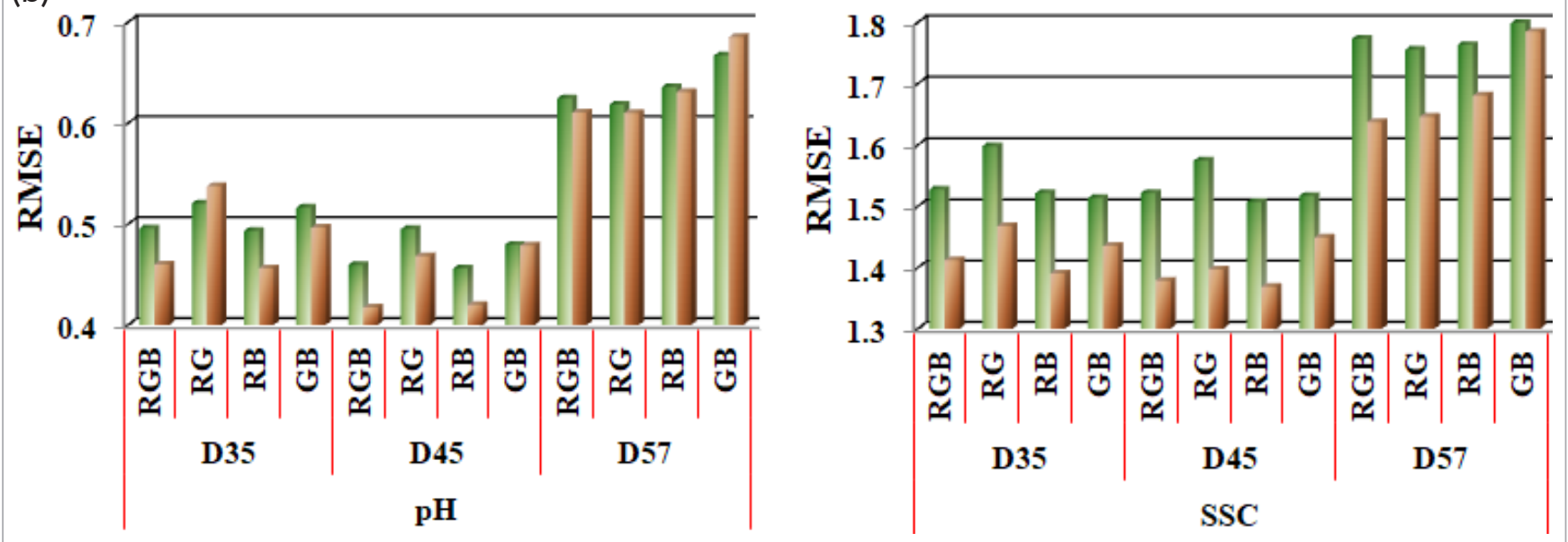

Figure 3. Overall measurement results represented in (a) correlation coefficient and (b) RMSE.

recorded for yellow-orange mango where higher LED CCT increases the brightness and yellow appearance of the mango. Figure 2(c i) shows the reflected visible LED spectra of the white diffuse reflectance surface in relation to the calibrated tungsten lamp spectrum. Figure 2 (c ii) and (c iii) shows the reflected white LED spectra from the surface of green and yellow-orange mango, respectively.

Since the measurement was calibrated using a tungsten halogen light source and a standard white diffuse surface as reference, the reflectance spectra imitate the illumination pattern of white LEDs, rather than the colour of the mangoes. From the graphs, it can be seen that peak reflectance at $550 \mathrm{~nm}$ for all spectra is much higher for LED D57 compared to D35 and D45. This is the main reason that $b^{*}$ tends towards a higher positive value.

For the prediction of mango $\mathrm{pH}$ and SSC using RGB and $M L R$, the combination between $R$ and $B$ for LED
D45 produced the best measurement accuracy with a correlation coefficient, $R=0.8630$ and root mean square error, $R M S E=0.4197 \mathrm{pH}$ for the $\mathrm{pH}$ validation dataset, and $R=0.7489$ and $R M S E=1.3685^{\circ}$ Brix for the SCC validation dataset. The best calibration algorithms for $\mathrm{pH}$ and SSC prediction are represented by Equation 1 and Equation 2, respectively.

$$
\begin{aligned}
& \mathrm{pH}=2.778+0.01493 \mathrm{R}-0.02139 \mathrm{~B} \\
& \mathrm{SSC}=8.077+0.03140 \mathrm{R}-0.0391 \mathrm{~B}
\end{aligned}
$$

The addition of $G$ to the prediction algorithm only improves the measurement accuracy by $1 \%$ or less. Figure 2(a) shows the comparative results between correlation coefficient for calibration and validation datasets using different combinations of R, $G$ and $B$ under illumination by different LED CCT. Figure 2(b) shows the respective values of RMSE for all measurements. Though LED D57 
may enhance the brightness and colour appearance of the fruit samples, for quantitative application, it recorded the lowest accuracy for all possible combination of $R, G$ and $\mathrm{B}$.

The quantitative experimental results obtained in this research are similar to the observation by Chiong and Omar, ${ }^{23}$ where white LED illumination with $4500 \mathrm{~K} \mathrm{CCT}$ produced the best visible spectroscopy measurement of mango quality (i.e. $\mathrm{pH}$ and SSC) and show a higher stability during calibration transfer between LEDs with different CCT.

\section{Conclusion}

The CCT of white LED has a noticeable influence on the appearance of fruits and in the quantitative analysis of fruit quality with the RGB technique. This study, which was conducted on Sala mangoes, has shown that higher CCT of white LED enhances the brightness and the colour appearance of the samples (i.e. green for immature mango and yellow for fully ripe mango). For pH and SSC measurement of mangoes using the RGB technique, white LED D45 with R and B components of the image produced the highest measurement accuracy for both $\mathrm{pH}$ and SSC. LED D57 produced the lowest measurement accuracy, despite its ability to enhance the colour appearance of mangoes. A similar experimental setup can be used to predict the qualities of an independent set of mango samples and even on different type of fruits, to further examine and understand the impact of white light CCT on the RGB images and subsequently on the accuracy of the developed quality measurement algorithms.

\section{References}

1. D.S. Prabha and J.S. Kumar, "Assessment of banana fruit maturity by image processing technique", J. Food Sci. Technol. 52, 1316-1327 (2015). doi: https:// doi.org/10.1007/s13197-013-1188-3

2. J. Asnor, S. Rosnah, Z. Wan and H. Badrul, "Pineapple maturity recognition using RGB extraction", World Academy of Science, Engineering and Technology 78, 147-150 (2013).

3. E. Guzmán, V. Baeten, J.A.F. Pierna and J.A. GarcíaMesa, "Determination of the olive maturity index of intact fruits using image analysis", J. Food Sci. Technol.
52, 1462-1470 (2015). doi: https://doi.org/10.1007/ s13197-013-1123-7

4. H.E. Balaguera-López and A. Herrera Arévalo, "Determining optimal harvest point for champa (Campomanesia lineatifolia R. \& P.) fruit based on skin color", Ingeniería e Investigación 32, 88-93 (2012).

5. G.B. Seymour, J.E. Taylor and G.A. Tucker, Biochemistry of Fruit Ripening. Springer, Netherlands (2012).

6. G. Seymour, G.A. Tucker, M. Poole and J. Giovannoni, The Molecular Biology and Biochemistry of Fruit Ripening. Wiley (2013).

7. P. Subedi and K.B. Walsh, "Assessment of sugar and starch in intact banana and mango fruit by SWNIR spectroscopy", Postharvest Biol. Technol. 62, 238245 (2011). doi: https://doi.org/10.1016/j.postharvbio.2011.06.014

8. A. Etienne, M. Génard, P. Lobit, D. Mbeguié-AMbéguié and C. Bugaud, "What controls fleshy fruit acidity? A review of malate and citrate accumulation in fruit cells", J. Exp. Bot. 64, 1451-1469 (2013). doi: https://doi.org/10.1093/jxb/ert035

9. R. Tharanathan, H. Yashoda and T. Prabha, "Mango (Mangifera indica L.), 'The king of fruits'-An overview", Food Rev. Int. 22, 95-123 (2006). doi: https:// doi.org/10.1080/87559120600574493

10. A.H. Gómez, Y. He and A.G. Pereira, "Nondestructive measurement of acidity, soluble solids and firmness of Satsuma mandarin using Vis/ NIR-spectroscopy techniques", J. Food Eng. 77, 313-319 (2006). doi: https://doi.org/10.1016/j. jfoodeng.2005.06.036

11. H. Zhang, J. Wang and S. Ye, "Predictions of acidity, soluble solids and firmness of pear using electronic nose technique", J. Food Eng. 86, 370-378 (2008). doi: https://doi.org/10.1016/j.jfoodeng.2007.08.026

12. S.-s. Wang, H.-n. Sun, J. Chen and A.-d. Sun, "Determination of soluble solids content of blueberries with near infrared spectroscopy", Sci. Technol. Food Ind. 2012(1), 340-341, 369 (2012).

13. A.F. Omar, "Spectroscopic profiling of soluble solids content and acidity of intact grape, lime, and star fruit", Sensor Rev. 33, 238-245 (2013). doi: https:// doi.org/10.1108/02602281311324690

14. J. Li, W. Huang, C. Zhao and B. Zhang, "A comparative study for the quantitative determination of soluble solids content, $\mathrm{pH}$ and firmness of pears by Vis/NIR spectroscopy", J. Food Eng. 116, 
324-332 (2013). doi: https://doi.org/10.1016/j. jfoodeng.2012.11.007

15. L.m. Yuan, L. Sun, J.r. Cai and H. Lin, "A preliminary study on whether the soluble solid content and acidity of oranges predicted by near infrared spectroscopy meet the sensory degustation", J. Food Proc. Eng. 38, 309-319 (2015). doi: https://doi. org/10.1111/jfpe.12104

16. M.S.J. Abarra, E.P. Serrano, V.C. Sabularse, H.E.T. Mendoza and E.J. del Rosario, "Determination of fruit ripeness degree of 'Carabao' mango (Mangifera indica L.) using digital photometry", Philippine J. Sci. 147, 249-253 (2018).

17. O.K.M. Yahaya, M.Z.M. Jafri, A.A. Aziz and A.F. Omar, "Determining Sala mango qualities with the use of RGB images captured by a mobile phone camera", AIP Conference Proceedings 1657, 060003 (2015). doi: https://doi.org/10.1063/1.4915190

18. Z. Xia, D. Wu, P. Nie and Y. He, "Non-invasive measurement of soluble solid content and $\mathrm{pH}$ in Kyoho grapes using a computer vision technique", Anal. Meth. 8, 3242-3248 (2016). doi: https://doi. org/10.1039/C5AY02694F
19. A.A. Mohamed and A.A. Shalaby, "Digital imaging devices as sensors for iron determination", Food Chem. 274, 360-367 (2019). doi: https://doi. org/10.1016/j.foodchem.2018.09.014

20. A. Klayann-Varejão-de Freitas Penha, C.S. Sousa and C.A. Oliveira, "A swift, easy and cheap protocol to evaluate the tooth bleaching in vitro", J. Clin. Exper. Dentistry 10(6), e579 (2018). doi: https://doi.org/ doi:10.4317/jced.54828

21. V.E. Nambi, K. Thangavel, S. Shahir and V. Geetha, "Evaluation of colour behavior during ripening of Banganapalli mango using CIE-Lab and RGB colour coordinates", J. Appl. Hort. 17(3), 205-209 (2015).

22. R.W.G. Hunt and E. Horwood, Measuring Colour, $2^{\text {nd }}$ Edn. Ellis Horwood, New York (1991).

23. W.L. Chiong and A.F. Omar, "White light emitting diode as potential replacement of tungstenhalogen lamp for visible spectroscopy system: a case study in the measurement of mango qualities", J. Instrum. 12, P07002 (2017). doi: https://doi. org/10.1088/1748-0221/12/07/P07002 